\title{
Décrire et comparer des formations d'ingénieurs interdisciplinaires : enjeux méthodologiques et épistémologiques
}

\author{
Anne-Sophie Godfroy ${ }^{1,2, a}$ \\ ${ }^{1}$ Sciences Normes Décision (SND)-www.snd-sorbonne.fr, Université Paris-Sorbonne - Paris IV, \\ CNRS : FRE3593 Rue Serpente, 75006 Paris, France \\ ${ }^{2}$ ESPE de Créteil UPEC-ESPE) - http: //espe.u-pec.fr, Université Paris Est Créteil, Rue Jean \\ Macé, 94380 Bonneuil-sur-Marne, France
}

\begin{abstract}
Résumé. La communication proposée s'appuie sur les résultats du projet européen HELENA (2009-2011) dont un objectif était la comparaison entre des formations d'ingénieur interdisciplinaires en Europe et leur réception par les étudiants dans huit pays. Le projet, reprenant les résultats d'un projet antérieur, définissait comme « interdisciplinaires » les formations qui comprenaient au moins 25 à $30 \%$ d'unités d'enseignement, exprimées en «crédits ECTS » (European Credit Transfer System), dans des disciplines autres que les disciplines scientifiques et techniques, notamment en sciences humaines et sociales. Il faisait l'hypothèse qu'une formation définie comme « interdisciplinaire » était davantage appréciée des étudiants qui la considéraient comme plus efficace pour les préparer à la vie professionnelle et plus attractive pour les étudiantes, encore peu nombreuses dans les écoles d'ingénieur. Les résultats de l'étude qui portait à la fois sur un décompte des crédits nécessaires à l'obtention du diplôme et sur des entretiens avec les étudiants, vérifient l'hypothèse dans certains pays, mais pas dans d'autres.

L'utilisation des « crédits ECTS » comme opérateur de commensurabilité était un choix méthodologique pertinent dans le cadre d'une comparaison européenne. Néanmoins, malgré l'utilisation de cet outil de mesure commun, la diversité des résultats obtenus met en évidence la complexité des réalités que recouvre le terme "disciplines autres que scientifiques et techniques » et les modalités de leur articulation à l'ensemble du curriculum. Elle conduit également à s'interroger sur le caractère "disciplinaire » au sens de la discipline académique, de nombreuses activités comme les stages ou les projets de fin d'études. Quel statut donner alors à ces activités ?

Une autre explication de cette variété des résultats vient de la diversité des formations d'ingénieurs elles-mêmes, qui reposent sur des cultures et des histoires différentes d'un pays à l'autre et dans un même pays. En outre, elles s'adressent à des étudiants aux profils très variés, et recouvrent des logiques de formation très hétérogènes, qui vont de la formation de l'hyper-spécialiste dans un domaine très technique à l'ingénieur-manager généraliste, en passant par toutes les logiques intermédiaires.

La communication reviendra sur les résultats du projet HELENA et sur leur interprétation dans une perspective heuristique. Un objectif de cette étude était de permettre une première comparaison afin de mettre en lumière les caractéristiques des enseignements
\end{abstract}

\footnotetext{
a e-mail : anne-sophie.godfroy@u-pec.fr
}

This is an Open Access article distributed under the terms of the Creative Commons Attribution License 4.0, which permits unrestricted use, distribution, and reproduction in any medium, provided the original work is properly cited. 
en école d'ingénieur et la complexité de leur organisation. Le projet a démontré l'efficacité des outils de mise en équivalence mis en place par le processus de Bologne. Il conviendrait dans un second temps de définir plus précisément les termes de la comparaison et de chercher des méthodologies comparatives plus élaborées de manière à mieux comprendre en quoi les sciences humaines et sociales préparent à la vie professionnelle ou rendent plus attractives des formations d'ingénieurs.

\section{Introduction : le projet HELENA et son contexte}

Cet article s'appuie sur les résultats du projet européen HELENA ${ }^{1}$ (2009-2011) dont un objectif était la comparaison entre des formations d'ingénieur interdisciplinaires en Europe et leur réception par les étudiants dans sept pays. En tant que chercheuse rattachée au cours de cette période au laboratoire «Sciences Techniques Éducation Formation» de l'École Normale Supérieure de Cachan, qui était partenaire du projet, j'étais en charge de la méthodologie, et ce sont les réflexions sur cette expérience qui ont nourri les contenus de cet article qui sont également présentées en anglais dans Gender and Interdisciplinary Education for Engineers (Béraud, Godfroy, \& Michel, 2012) et dans l'article « Thinking Interdisciplinarity in Engineering Education: Challenges for Future Research » (Godfroy, 2012).

L'attractivité des formations d'ingénieurs est considérée par la Commission Européenne comme une condition essentielle du développement économique et technique de l'Union Européenne (Mariano Gago, 2004), (European Commission, 2010). Certaines études indiquent que le manque d'interdisciplinarité dans les programmes de formation d'ingénieurs expliquerait la faible attractivité de ces formations. Le projet INDECS ${ }^{2}$ (INDECS Project Final Report: Potentials of interdisciplinary degree courses in engineering, information technology, natural and socio-economics sciences in a changing society, 2002) avait étudié en 2002 des études de cas de formations d'ingénieurs interdisciplinaires et avait confirmé que, dans certaines conditions, la présence des disciplines autres que scientifiques et techniques et la prise en compte des questions sociétales augmentait l'attractivité des formations techniques, notamment envers les étudiantes (Beraud, 2003). Ce lien entre l'attractivité et l'interdisciplinarité est également souligné par le projet WOMENG (Creating cultures of success for women engineers - Womeng, 2006) qui avait montré que 34,6\% des étudiants ingénieurs et 37,9\% des étudiantes souhaitaient davantage d'interdisciplinarité dans leur formation, c'est-à-dire davantage d'humanités et de sciences humaines et sociales, et que d'autre part, que $26,9 \%$ des étudiants qui n'avaient pas choisi des formations d'ingénieurs, alors qu'ils auraient pu le faire, auraient changé d'avis si les programmes avaient comporté plus de sciences sociales et d'humanités (Creating cultures of success for women engineers - Womeng, 2006) (Waechter, 2005). Dans ce contexte, le projet HELENA tentait de questionner et de confirmer ces conclusions en élargissant l'échantillon sur lequel les hypothèses étaient testées. En effet, les projets précédents portaient sur des échantillons assez réduits (des études de cas) alors qu'HELENA avait l'ambition de recenser l'ensemble des formations d'ingénieur dans deux domaines par pays, ou, pour les très grands pays (France, Royaume-Uni, Allemagne) au moins le tiers des formations. Cette approche à plus grande échelle s'accompagnait d'une tentative de quantification de la part d'interdisciplinarité dans ces formations de façon à rechercher des corrélations entre la proportion d'interdisciplinarité et la proportion d'étudiantes dans les formations, car la question de l'attractivité envers les étudiantes, bien que très peu développée dans cet article, était

\footnotetext{
1 Titre complet du projet: Higher Education Leading to Engineering and Scientific Careers, acronyme « HELENA ». Les institutions partenaires du projet étaient l'École Normale Supérieure de Cachan en France, l'Université de Siauliai en Lituanie qui assurait la coordination, l'Université de Loughborough au Royaume-Uni, l'entreprise Tecnalia en Espagne, l'Université de Klagenfurt en Autriche, l'Institut Pupin en Serbie et l'association ECEPIE en France. Ce projet a été financé par la Commission Européenne dans le cadre du $7^{e}$ programme cadre de recherche et de développement (PCRD) pour une durée de 30 mois, de 2009 à 2011. Site du projet : http: //www . fp7-helena.org/

2 INDECS : "Potentials on Interdisciplinary Degree Courses in Engineering, Information Technology, Natural and SocioEconomic Sciences in a Changing Society”, projet financé par l’Union Européenne 2001-2002.
} 
également l'un des objectifs du projet. Nous expliquerons plus loin en quoi a consisté ce travail de quantification.

Plus précisément, un des objectifs du projet HELENA était de vérifier ou infirmer les deux hypothèses suivantes : la première était qu'un enseignement «interdisciplinaire », c'est-à-dire un programme de formation qui associait des cours dans des disciplines scientifiques et techniques, traditionnelles en école d'ingénieurs, et des disciplines comme les sciences humaines et sociales, souvent associées à une pédagogie de projet $^{3}$ et de groupe était plus attractif pour les étudiants et en particulier pour les étudiantes ; la seconde était que les étudiantes réussissaient mieux dans ces programmes interdisciplinaires. Nous reviendrons longuement au cours de cet article sur la définition donnée au concept d'interdisciplinarité dans le cadre d'HELENA.

Les méthodologies retenues par le projet HELENA pour répondre à ces deux hypothèses combinaient d'une part une enquête extensive sur les formations d'ingénieurs existantes, d'inspiration quantitative (dénombrement des formations dans chaque pays dans deux spécialités, l'une où la proportion d'étudiantes était importante, l'autre où elle était particulièrement faible, puis décompte du nombre de crédits ECTS dits « interdisciplinaires », nous reviendrons sur cette définition), et d'autre part des approches qualitatives sur quelques cas dans chaque pays séries d'entretiens avec des étudiants, analyse détaillée des maquettes, entretiens avec les concepteurs de la formation, etc.). L'étude des formations devait en outre permettre de dégager ce que l'interdisciplinarité changeait à l'attractivité.

Cet article se propose de présenter et décrire la méthodologie et les résultats d'HELENA, de questionner le concept d' « interdisciplinarité » dans les formations d'ingénieurs et de proposer des questions de recherche à venir.

\section{La méthodologie du projet HELENA}

Le projet HELENA a volontairement utilisé un concept pragmatique et provisoire pour définir l'interdisciplinarité et laissé de côté, au moins pour la partie quantitative du projet, une réflexion plus approfondie sur l'interdisciplinarité (voir par exemple (Paulsen, 2008) (Maingain \& Dufour, 2002) (Vinck, 2001). Il s'agissait de «commencer quelque part», en étant pleinement conscients des limites de la définition retenue mais en recherchant un moyen simple de « quantifier » la part d'interdisciplinarité des formations. Il fallait en outre choisir un concept qui se prêterait à la comparaison européenne et donc retenir une unité commune. Dans cette perspective, la solution la plus simple en première approche était de retenir comme unité commune les crédits $\mathrm{ECTS}^{4}$ puisque tous les programmes de formation européens ont été traduits dans cette unité. Chaque programme de formation a donc été examiné, et chaque cours de chaque programme, à partir de sa description a été qualifié de « traditionnel » ou « interdisciplinaire ». Les cours qui relevaient des disciplines scientifiques et techniques ont été qualifiés de «traditionnels », les cours dans des disciplines autres que les sciences et techniques d' « interdisciplinaires », enfin les cours qui se référaient à plusieurs disciplines différentes, scientifiques et techniques ou non, ont été également qualifiés d' «interdisciplinaires ».

Cette approche manquait de consistance conceptuelle mais avait le mérite de reprendre les approches spontanées de nombreuses formations d'ingénieurs qui distinguent d'un côté les sciences de l'ingénieur

\footnotetext{
3 On entend par là des enseignements articulés autour d'un projet qui sert de support aux activités des étudiants, en groupe ou individuellement. Ces projets sont ancrés dans des situations concrètes à partir desquelles les étudiants doivent construire leur activité, acquérir de connaissances, faire des choix techniques, interagir avec l'environnement du projet, etc. Selon les cas, il s'agit d'un projet long (par exemple le projet de fin d'études) ou de projets très ponctuels. L'élément commun à cette approche est l'ancrage dans un contexte concret avec sa complexité et la liberté laissée aux étudiants de construire leur problématique et leur approche, en l'explicitant.

${ }^{4}$ European Credit Transfert System. Un système de crédits est une méthode qui permet d'attribuer des crédits à toutes les composantes d'un programme d'études. La définition des crédits au niveau de l'enseignement supérieur se base sur les paramètres suivants : charge de travail de l'étudiant, nombre d'heures de cours et objectifs de formation. Source http://www.europeeducation-formation.fr/page/ects
} 
et les disciplines académiques scientifiques jugées comme «traditionnelles » dans ces formations, à savoir les mathématiques, la physique, la chimie, éventuellement la biologie, et de l'autre, tout ce qui ne correspond pas à cette définition et regroupe indifféremment les langues, les sciences humaines et sociales, le droit, l'économie, les activités physiques et sportives, parfois les stages, etc. Ces activités « autres » sont également parfois qualifiées d' «interdisciplinaires ». Si dans certains pays comme la France, le décompte s'est fait à la main, dans d'autres comme l'Autriche, le regroupement des cours « autres » sous la catégorie « interdisciplinaire » était déjà présent dans les descriptifs de formation.

Dans certains cas, comme les cours « interdisciplinaires » sont pensés par opposition aux cours « traditionnels », ils sont parfois qualifiés d' « innovants». Le manque de consistance conceptuelle de ces définitions a rendu le processus de comptage des ECTS «traditionnels » et «interdisciplinaires » délicat, mais a fourni un point de départ opérationnel intéressant et a permis de mettre à jour cette absence de consistance même là où la classification était déjà présente.

Le parti pris de cette étude était de travailler en extension et de trouver une première approche quantitative, une autre option complémentaire aurait été de travailler en détail les concepts d'inter-, trans- et pluridisciplinarité et de rentrer dans le détail des programmes de formation, les approches pédagogiques et les interactions entre les différents cours. Cette seconde option n'était pas possible dans le cadre de ce projet mais pourrait être au cœur d'un projet futur.

Dans chacun des pays partenaires, le projet HELENA a examiné toutes les formations dans deux domaines d'études, l'un où la proportion d'étudiante était très faible, l'autre où la proportion d'étudiante était très élevée (par exemple l'informatique et le génie civil dans le cas de la France). Étant donné le critère retenu, les domaines d'études n'étaient pas toujours les mêmes d'un pays à l'autre, mais pour chaque domaine, plusieurs cas ont pu être observés, dans différents pays ou un seul (voir infra). Dans le cas des très grands pays comme l'Allemagne, la France ou le Royaume-Uni, une étude de toutes les formations dans un domaine était impossible faute de temps. Dans ces cas, on a étudié au moins le tiers des formations existantes pour les domaines choisis, ce qui représentait déjà un échantillon important.

Les crédits ECTS «traditionnels » et «interdisciplinaires » ont été décomptés pour chaque programme d'étude. Quand un programme comprenait plus de $25 \%$ d'ECTS « interdisciplinaires », le programme était qualifié « interdisciplinaire ». Ce seuil reprenait les résultats du projet INDECS qui avait montré, sur des études de cas, que les cours « autres » que les sciences de l'ingénieur et les disciplines scientifiques qui leur étaient habituellement associé avaient un effet sur le recrutement et la motivation des étudiants à partir d'une présence significative de $25 \%$ des crédits au moins dans la formation (Beraud, 2003).

La proportion d'étudiantes en première année, en moyenne, parmi les diplômés, était également calculée dans chaque programme, ainsi que la corrélation éventuelle entre cette proportion et le caractère interdisciplinaire ou traditionnel de la formation, puisque la mesure de l'attractivité envers les étudiantes était une des préoccupations centrales du projet.

Au total 189 formations dans 7 pays ont été étudiées dans les domaines suivants : l'informatique (Autriche, France, Macédoine, Serbie, Espagne), le génie civil (France et Royaume-Uni), le génie environnemental (Lituanie), le génie mécanique (Lituanie) et le management industriel (Espagne). L'enquête a qualifié 32 formations d' « interdisciplinaires» et 157 de « traditionnelles ». Le degré d'interdisciplinarité variait considérablement, de $0 \%$ (Autriche, Serbie) à $48 \%$ (Espagne : UAB Engineering Management). Aucune corrélation entre la proportion d'étudiantes et le degré d'interdisciplinarité n'a été observée pour l'ensemble des pays, elle est vérifiée dans certains mais pas dans d'autres.

En complément de cette étude quantitative, des études de cas qualitatives ont été menées pour étudier plus en détail les contenus des programmes et les représentations des étudiants. Parmi les 189 formations étudiées, il s'agissait dans chaque pays de retenir quatre programmes de formations et de réaliser à chaque fois une étude de cas qui comportait plusieurs volets : l'analyse des programmes, l'analyse des plaquettes de présentation et des sites internet, et des entretiens avec des étudiants et des professeurs. Il 
s'agissait de confronter dans la mesure du possible l'observation des contenus effectifs des programmes, l'image que souhaitait donner l'institution et son discours sur ses choix pédagogiques à travers son matériel de promotion, et la manière dont les programmes étaient reçus et analysés par les étudiants, puis d'analyser ou la cohérence ou les décalages entre ces différents corpus.

Le choix des cadre études de cas reprenait celui des deux domaines de formation retenus au départ dans chaque pays, avec pour chaque domaine, l'étude d'un programme de formation traditionnel (parmi les plus basses proportions de crédits ECTS « interdisciplinaires ») et celle d'un programme de formation «interdisciplinaire » (parmi les plus hautes proportions de crédits ECTS « interdisciplinaires »). Entre des formations équivalentes au regard de ces critères, le choix final s'est fait en fonction de l'environnement de chaque partenaire, notamment de la proximité géographique et des facilités d'accès aux institutions étudiées.

Au total 24 études de cas ont été réalisées. Les disciplines « interdisciplinaires » les plus fréquentes ont été le management, business, l'économie, les langues et plus rarement la philosophie, l'histoire, le design, la sociologie, les sciences de l'environnement avec une forte composante sociétale. Puisque les questions de genre étaient également au cœur du projet - même si elles sont très peu abordées dans cet article - les cours sur le genre ont été particulièrement étudiés, mais seuls deux cours de ce type ont été rencontrés parmi les 24 études de cas, dans les formations « interdisciplinaires » autrichiennes de Linz et de Klagenfurt.

162 entretiens semi-directifs ont été conduits, 72 avec des étudiants et étudiantes de formations « traditionnelles », 90 avec des étudiants et étudiantes de formations « interdisciplinaires », sur les 162 entretiens, on dénombre 79 entretiens avec des étudiantes et 83 avec des étudiants.

En ce qui concerne la France, nous avions délibérément choisi des formations de qualité, mais sans notoriété extrêmement forte, à l'exception peut-être de l'INSA-Lyon. On entend par là des formations qui ne recrutent pas exclusivement ou presque leurs étudiants après une classe préparatoire, et qui ne sont pas très anciennes (par contraste avec les plus anciennes écoles créées au XVIIIe ou au XIXe siècles). En effet, alors que les grandes écoles les plus prestigieuses, qui répondent à ces deux critères, sont souvent étudiées, les formations d'ingénieur plus récentes sont assez peu étudiées et souvent très innovantes, notamment dans leur offre de formation et leurs choix pédagogiques, d'où l'intérêt de notre choix. En effet, si les écoles les plus anciennes et difficiles, en terme de sélectivité du concours d'entrée, ont peu d'efforts à faire pour attirer d'excellents étudiants sur leur réputation réelle ou supposée, les écoles moins reconnues doivent argumenter davantage pour attirer des étudiants qui ont le choix entre plusieurs écoles dans lesquelles ils ont été acceptés. Néanmoins, d'autres choix ont parfois été faits dans les autres pays, ce qui peut créer des distorsions dans la comparaison internationale.

\section{Les principaux résultats du projet HELENA}

Le corpus a-t-il permis de vérifier les hypothèses de départ? De très nombreux articles de recherche soutiennent ces hypothèses, cependant, comme Christine Waechter l'a montré (Waechter, 2012), dans l'échantillon du projet HELENA l'hypothèse que les formations «interdisciplinaires » ont une plus grande proportion d'étudiantes est vérifiée en Autriche, en Espagne, en Lituanie, partiellement en Serbie (sur de trop petits nombres), mais invalidée au Royaume-Uni et en France. En ce qui concerne la seconde hypothèse, à savoir que les étudiantes réussissent mieux dans les formations « interdisciplinaires », elle est vérifiée en Espagne et en Lituanie, elle correspond à une tendance en Autriche, elle est fausse ailleurs. On constate également que les formations créées après le processus de Bologne tendent à proposer davantage de cours « interdisciplinaires » mais que les variations sont très importantes d'un pays à l'autre. Elles peuvent s'expliquer par des histoires de l'enseignement de l'ingénierie très différentes, des traditions et des points du vue différents sur ces enseignements, mais aussi par des manière de compter les ECTS insuffisamment harmonisées.

Anita Thaler a résumé dans un article (Thaler, 2012) les principaux résultats des entretiens avec les étudiants. Les étudiants des formations «interdisciplinaires » sont plus enthousiastes que ceux 
Tableau 1. Récapitulatif des études de cas dans chaque pays. Entre parenthèse, le degré d'interdisciplinaruté en \%.

\begin{tabular}{|c|c|c|c|c|}
\hline & $\begin{array}{l}\text { Traditional } \\
\text { case study } 1\end{array}$ & $\begin{array}{l}\text { Innovative } \\
\text { case study } 1\end{array}$ & $\begin{array}{r}\text { Traditional } \\
\text { case study } 2\end{array}$ & $\begin{array}{l}\text { Innovative } \\
\text { case study } 2\end{array}$ \\
\hline Austria & $\begin{array}{l}\text { Vienna University of } \\
\text { Technology: } \\
\text { Media informatics } \\
(11 \%)\end{array}$ & $\begin{array}{l}\text { Alpen-Adria-Universität } \\
\text { Klagenfurt: } \\
\text { Information management } \\
(42 \%)\end{array}$ & $\begin{array}{l}\text { Graz University of } \\
\text { Technology: } \\
\text { Software development } \\
\text { + business } \\
\text { management }(19 \%)\end{array}$ & $\begin{array}{c}\text { Johannes Kepler } \\
\text { University Linz: } \\
\text { Business informatics } \\
(35 \%)\end{array}$ \\
\hline France & $\begin{array}{c}\text { EP Grenoblè: } \\
\text { IT networks }+ \\
\text { multimedia } \\
\text { communications }(22 \%)\end{array}$ & $\begin{array}{c}\text { EP Orléans: } \\
\text { Civil engineering }(22 \%)\end{array}$ & \begin{tabular}{|c|} 
EP Grenoblè: \\
Industrial data \\
processing and \\
instrumentation $(15 \%)$
\end{tabular} & \begin{tabular}{|c|} 
INSA Lyon: \\
Civil engineering and \\
urban planning $(20 \%)$
\end{tabular} \\
\hline Lithuania & $\begin{array}{l}\text { Kaunas University of } \\
\text { Technology: } \\
\text { Mechanical engineering } \\
(20 \%)\end{array}$ & $\begin{array}{l}\text { Kaunas University of } \\
\text { Technology: } \\
\text { Environmental } \\
\text { engineering }(25 \%) \\
\end{array}$ & $\begin{array}{c}\text { Siauliai University: } \\
\text { Mechanical } \\
\text { engineering }(22 \%)\end{array}$ & $\begin{array}{l}\text { Siauliai University: } \\
\text { Environmental and } \\
\text { professional safety } \\
(34 \%)\end{array}$ \\
\hline Macedonia & - & - & $\begin{array}{c}\text { Ss. Cyril and } \\
\text { Methodius University: } \\
\text { Computer system } \\
\text { engineering and } \\
\text { automation }(8-19 \%) \\
\end{array}$ & $\begin{array}{c}\text { Ss. Cyril and } \\
\text { Methodius University: } \\
\text { Informatics and } \\
\text { computer engineering } \\
(4-14 \%) \\
\end{array}$ \\
\hline Serbia & $\begin{array}{c}\text { University of Belgrade: } \\
\text { Computer engineering } \\
\text { and informatics }(0-18 \%)\end{array}$ & $\begin{array}{l}\text { University of Belgrade: } \\
\text { Information systems and } \\
\text { technologies }(30 \%)\end{array}$ & - & - \\
\hline Spain & $\begin{array}{c}\text { UPC Barcelona Tech, } \\
\text { Polytechnic University of } \\
\text { Catalunya: } \\
\text { ICT engineering }(13 \%)\end{array}$ & $\begin{array}{c}\text { MU Mondragon } \\
\text { University: } \\
\text { ICT engineering (30\%) }\end{array}$ & \begin{tabular}{|} 
UPV-EHU Basque \\
Country University: \\
Industrial management \\
engineering $(23 \%)$ \\
\end{tabular} & \begin{tabular}{|c|} 
MU Mondragon \\
University: \\
Industrial management \\
engineering $(33 \%)$ \\
\end{tabular} \\
\hline UK & $\begin{array}{l}\text { University of Liverpool: } \\
\text { Civil engineering }(16 \%)\end{array}$ & \begin{tabular}{|c|} 
Loughborough \\
University: \\
Civil engineering $(19 \%)$
\end{tabular} & $\begin{array}{l}\text { Coventry University: } \\
\text { Civil engineering* } \\
(28 \%)\end{array}$ & $\begin{array}{c}\text { University of } \\
\text { Warwick: } \\
\text { Civil engineering } \\
(36 \%)\end{array}$ \\
\hline
\end{tabular}

(NB: pour le Royaume-Uni, le $3^{e}$ cas, avec $28 \%$ d'interdisciplinarité, a été retenu dans la catégorie « interdisciplinaire»).

Source: (Thaler, 2012).

des formations « traditionnelles » qui se plaignent du manque d'expérience pratique. Les étudiants des formations «interdisciplinaires » sont également plus satisfaits en général (en Autriche et en Macédoine), et particulièrement satisfaits de la pédagogie (en Espagne). Ils pensent qu'ils ont de meilleures perspectives d'emploi (en Autriche et en France). Enfin, les étudiants des formations « interdisciplinaires » se voient en « managers » (en Autriche et en Serbie) alors que ceux des formations « traditionnelles » se voient plus souvent en « ingénieurs ». Par ailleurs, certains étudiants n’ont pas choisi leur programme de formation seulement pour son contenu, ils ont aussi choisi une université ou une école pour sa réputation. Par exemple, un étudiant espagnol a déclaré : «J'ai toujours voulu étudier l'informatique. Quand j'ai dû décider quelle université choisir, j'ai choisi celle-ci car elle était prestigieuse et avait une bonne réputation ». La réputation est également souvent soulignée en France, au Royaume-Uni, en Macédoine, en Serbie et en Lituanie.

Il est difficile de résumer les résultats de l'étude en quelques pages, on se reportera pour un aperçu plus complet au volume Gender and Interdisciplinary Education for Engineers (Béraud et al., 2012) qui comprend plusieurs contributions sur les résultats du projet HELENA.

Ces résultats sont complexes, parfois inattendus, et peuvent s'interpréter de la manière suivante : quand le contenu des programmes est le premier critère de choix, l'introduction de davantage 
d'interdisciplinarité renforce l'attractivité des formations et l'hypothèse est vérifiée. Néanmoins, si le critère de choix n'est pas d'abord le contenu de la formation, l'interdisciplinarité a peu d'incidence sur l'attractivité. Le classement, le prestige, le hasard parfois, les raisons personnelles ou les stéréotypes passent avant les contenus dans le choix des étudiants. Les procédures d'admission sur un concours ou un score reflètent moins les choix personnels. Cependant, même dans ce cas, dans les entretiens avec les étudiants, l'attractivité de l'interdisciplinarité réapparait au cours de la formation, quand les étudiants choisissent des options et des spécialités.

Autre résultat du projet HELENA important à approfondir: les cours « interdisciplinaires » ne sont attractifs que s'ils sont pris au sérieux par les concepteurs de la formation, c'est-à-dire s'ils font partie d'un curriculum cohérent et intégré, s'ils sont enseigné par des professeurs qualifiés et permanents avec une pédagogie appropriée. Le seul message publicitaire, exprimé dans les plaquettes de présentation de l'école et sur son site internet, ne suffit pas. Si le discours de l'institution ne correspondent pas à la réalité de son offre pédagogique, les entretiens avec les étudiants montrent qu'ils ne sont pas dupes et jugent sévèrement le décalage entre l'image et la réalité de la formation. L'effet produit est alors probablement inverse de l'effet attendu.

\section{Les questions sur l'interdisciplinarité soulevées par HELENA}

Au-delà du projet HELENA, la méthodologie développée à cette occasion nous semble un point de départ intéressant pour penser plus généralement la question de l'interdisciplinarité et de la place des sciences humaines et sociales dans les écoles d'ingénieurs qui était l'un des thèmes du colloque « Sciences humaines en sciences et techniques 2013 ».

Le HELENA a mis en œuvre une définition provisoire et pragmatique de l'interdisciplinarité afin de permette la comparaison facile de programmes de formation à travers l'Europe. Dans cette perspective le choix du dénombrement des ECTS était efficient, même si, comme nous l'avons déjà souligné, la correspondance entre un titre et une courte description de cours avec une caractérisation comme « interdisciplinaire» ou «traditionnel» manque de consistance conceptuelle et met en relief des questions de recherche à explorer dans des études à venir. Des listes avaient été constituées par les différentes équipes qui avaient répertorié ce qu'elles considéraient comme des disciplines « autres que scientifiques et techniques ».

En pratique, le concept d'interdisciplinarité, ainsi défini, s'est révélé un concept efficace quand le concept était déjà intégré à la conception du curriculum, dans ce cas, le décompte des crédits ECTS était simple et signifiant comme dans le cas de l'Autriche.

Dans les autres cas, il s'agissait d'un concept très flou qui pouvait signifier tantôt deux ou plusieurs disciplines liées dans un même cours ou des disciplines inhabituelles dans une formation donnée, par exemple des disciplines autres que scientifiques et techniques dans une formation d'ingénieur. Cette définition négligeait un travail sérieux sur le concept de « discipline » et sa construction historique. Parmi les nombreux ouvrages sur la questions, on peut citer Chaos of Disciplines. (Abbott, 2001) qui met en évidence le caractère à la fois fortement construit et instable de ce que sont les disciplines académiques. Outre l'impasse sur le concept même de «discipline », cette définition négligeait également une réflexion sur approfondie sur « multi », «pluri », « inter », « trans » disciplinaire (Origgi \& Darbellay, 2010) (Heintz, Origgi, \& Sperber, 2004) et la prise en compte de l'articulation des différents cours en un tout cohérent (Maingain \& Dufour, 2002).

Pour aller au delà du travail d'exploration entrepris par HELENA, il serait nécessaire de se donner a minima une définition claire des disciplines et de ce qu'est une discipline. Pour être complet, il faudrait y ajouter l'histoire et la dynamique des disciplines (voir par exemple (Collectif, 1988). Abbot (Abbott, 2001) a montré dans Chaos of Disciplines comment les disciplines des sciences sociales ne sont pas des entités stables mais des constructions intellectuelles qui changent au cours du temps, qui apparaissent, disparaissent, se rassemblent ou divergent en fonction d'éléments à la fois intellectuels, politiques et 
conjoncturels. Le même travail serait à intégrer à notre réflexion pour les disciplines enseignées dans les écoles d'ingénieurs.

Au-delà de ces définitions conceptuelles, une meilleure prise en compte du contexte académique et de ses implications serait nécessaire pour comprendre les enjeux portés par telle ou telle forme d'interdisciplinarité. En effet, chaque type de formation a ses spécificités, ses disciplines centrales, son histoire, ses contraintes propres, et l'interdisciplinarité, sous ses multiples formes, n'est pas reçue de la même façon selon. Par exemple, une langue vivante n'a pas le même statut dans une école d'ingénieurs et dans une faculté de Lettres et Sciences Humaines, les mathématiques peuvent être perçues comme une discipline sélective ou un rite de passage ou comme une discipline très facultative selon le contexte des différentes formations. Des remarques similaires pourraient être faites pour toutes les disciplines. A l'intérieur même des écoles d'ingénieurs, des contraintes spécifiques peuvent travailler les maquettes de formation, on renverra sur ce sujet à la deuxième partie de ce recueil et notamment à l'article de Michel Cotte $^{5}$.

Une question plus complexe est posée par les contenus a-disciplinaires ou au moins difficilement assimilables à une discipline au sens académique et des choix pédagogiques qui leur sont associés. Nous nous sommes interrogés sur la manière dont il convenait de caractériser les projets de fin d'études, les mémoires et les stages. Relèvent-ils d'une discipline universitaire ? Sont-ils autre chose qu'une modalité pédagogique ? Doit-on les considérer comme pluridisciplinaires ou traditionnels ? On serait tenté de répondre en fonction du contenu des stages et des mémoires, mais dans ce cas, le programme changerait de caractère avec chaque étudiant. Dans le cas des formations françaises, le fait de qualifier de «pluridisciplinaire » le projet de fin d'études qui représente la moitié des ECTS de la deuxième année de master faisait basculer pratiquement toutes les formations dans la catégorie «interdisciplinaire », l'enjeu est donc très important. Peut-être faudrait-il réserver une catégorie particulière pour ces contenus ancrés dans une expérience de terrain et difficiles à qualifier en termes académiques.

L'autre enseignement à tirer de la méthodologie d'HELENA, directement liée au choix d'examiner est très grand nombre de programmes, est que la prise en compte de l'interdisciplinarité quantitative ne suffit pas, il faut prendre en compte également la manière dont les cours "interdisciplinaires » sont enseignés et articulés à l'ensemble du curriculum, s'interroger sur leur structure et sur l'identité des professionnels chargés de les enseigner. Certes, HELENA abordait indirectement cette question dans les études de cas à travers des entretiens avec des étudiants et les concepteurs de la formation, mais la question mériterait d'être approfondie et systématisée. Les enseignants peuvent être des enseignantschercheurs, permanents ou pas, ou des intervenants issus du monde professionnels, situations qui ont des implications importantes pour l'intégration de ces cours dans un curriculum cohérent. Enfin, la pédagogie proposée par ces cours est également un élément important comme l'ont montré plusieurs études (Kourilsky \& Tellez, 2003) (Maingain \& Dufour, 2002). Dans le projet HELENA, les entretiens avec les étudiants ont permis de collecter des informations, mais ces sujets devraient être étudiés de manière plus systématique.

Enfin, la réflexion sur l'interdisciplinarité ne peut pas manquer de rencontrer la réflexion le nouveau mode de production des savoirs proposée par Nowotny, Scott et Gibbons. (Nowotny, Scott, \& Gibbons, 2001) qui caractérisent précisément le nouveau mode de production des savoirs, le « mode 2 » comme « interdisciplinaire ». Dans cette hypothèse l'enseignement des sciences et techniques ne ferait que suivre l'évolution du mode de production des sciences et techniques. Un enseignement « interdisciplinaire » ne serait pas seulement une question d'attractivité pour les étudiants, mais un changement de paradigme absolument nécessaire pour mettre à jour l'enseignement des sciences et techniques et le faire correspondre aux sciences et techniques qui se font. A cet égard, on constate que

\footnotetext{
${ }^{5}$ La partie 2 de ce volume, «Influences du contexte » donne de nombreux exemples de l'importance du contexte académique dans la construction des formations.
} 
la question des méthodes et des contenus d'enseignement sont ici étroitement liés, et demanderaient là encore une enquête plus poussée.

\section{Quelques réflexions suscitées par le projet HELENA}

Nous renvoyons au volume publié sur le projet HELENA et sa confrontation à d'autres recherches pour un exposé détaillé des résultats du projet (Béraud et al., 2012). Ce qui nous intéresse dans le contexte de cette contribution est de souligner les questions soulevées par la méthodologie du projet HELENA, son intérêt heuristique, et de les mettre en relation avec la problématique du colloque « sciences humaines en sciences et techniques ». Nous avons retenu trois questions fondamentales et intimement liées que le projet soulève et permet d'aborder de manière originale : l'attractivité des formations, les identités des ingénieurs et les parcours étudiants et professionnels.

\subsection{Interdisciplinarité et attractivité des formations}

Face à la désaffection pour les études scientifiques et techniques préoccupante dans de nombreux pays, de nombreuses études se sont préoccupées de l'attractivité des formations scientifiques et techniques et l'abordent en général sous l'angle de l'attitude et des perceptions des étudiants face à ces disciplines et ces formations (Kaptan \& Timurlenk, 2012). Jonathan Osborne, Shirley Simon et Sue Collins ont proposé un panorama de la littérature sur ce sujet (Osborne, Simon, \& Collins, 2003). Cette problématique rejoint les objectifs d'HELENA qui se proposait de travailler sur l'attractivité des formations d'ingénieurs, et plus particulièrement l'attractivité envers les étudiantes. Cette problématique est abordée dans HELENA par Thaler (Thaler, 2012) à travers les entretiens avec les étudiants et étudiantes, ce qui rejoint les méthodologies énumérées par Osborne et alii.

Les résultats du projet laissent néanmoins entrevoir d'autres manières de définir et d'aborder « l'attractivité » et de relier cette question de façon originale à celle de l'interdisciplinarité des formations. Dans ce contexte, il importe de souligner que cette nouvelle définition de l'attractivité, par d'autres approches que les perceptions des étudiants, n'est pas toujours stable. En effet, comment définir l'attractivité? Par un nombre d'étudiants, par la proportion entre étudiants et étudiantes, par les bons taux de réussite des étudiants et/ou des étudiantes, mesures qui permettraient toutes de quantifier l'attractivité, ou plus classiquement par l'appréciation positive des étudiants ou des étudiantes ? L'attractivité se mesure-t-elle par rapport à autre chose qui serait moins attractif, dans ce cas à par rapport à quoi ?

HELENA a montré les nombreuses interférences entre l'interdisciplinarité et d'autres facteurs d'attraction, par exemple le prestige ou la notoriété des formations, le classement des formations (quand il existe) ou encore le classement dans étudiants aux concours d'entrée, les perspectives d'emploi, l'intérêt personnel, etc. Selon les contextes, un facteur prime sur les autres. La dynamique complexe de l'attractivité reste à décrire en gardant à l'esprit que les corrélations ne doivent pas être confondues avec les relations de causalité. Au cours de la période récente, l'université a connu des mutations importantes avec la mise en œuvre du processus de Bologne, un accès démocratisé aux études supérieures et une plus grande mobilité internationale (programmes ERASMUS, possibilités de doubles masters européens etc.). Les effets de ces changements profonds doivent être également pris en considération pour comprendre les relations complexes entretenues par l'interdisciplinarité et l'attractivité. Un fort degré d'interdisciplinarité est-il toujours corrélé à une forte attractivité de la formation ? HELENA démontre que cette corrélation est à interpréter dans un contexte plus large qui prend en compte les modalités d'accès et de choix de la formation, les stratégies personnelles des étudiants dans un environnement internationalisé (sur lesquelles nous reviendront plus bas), les stratégies des institutions elles-mêmes, qui choisissent un positionnement dans l'offre de formation et font le choix de recruter certains profils d'étudiants, enfin les cultures et histoires nationales qui valorisent plus ou moins certains domaines de formation et certaines institutions. 
Le souci d'améliorer l'attractivité des formations d'ingénieurs vis-à-vis des étudiantes en y introduisant davantage d'interdisciplinarité souligne également un risque rarement aperçu, celui de « genrer » les disciplines et de tomber dans des stéréotypes: les « soft skills » et les sciences humaines et sociales seraient féminines et attireraient les étudiantes alors que les sciences dites « dures » seraient masculines. «Adoucir » la formation en y introduisant des «soft skills » attirerait les étudiantes. Les raisons d'introduire de l'interdisciplinarité dans les formations d 'ingénieurs et d'expliquer en quoi ces nouvelles formations sont attractives doivent éviter ce type d'explication stéréotypées et mener une réflexion sérieuse sur l'interdisciplinarité et les constructions disciplinaires avec leur histoire.

\subsection{Penser la question de l'interdisciplinarité dans les formations en relation avec les identités des ingénieurs}

Nous ne devons pas non plus nous dissimuler les enjeux politiques et économiques qui sont présents derrière l'injonction d'attractivité. Comme le soulignent les rapports de la Commission Européenne (Mariano Gago, 2004) (European Commission, 2010), il n'y aurait pas assez d'ingénieurs pour soutenir un développement technologique créateur de forte valeur ajoutée et donc de prospérité, il faudrait donc augmenter l'attractivité de ces carrières et augmenter le vivier de recrutement, notamment en stimulant le développement d'un vivier d'étudiantes, encore peu nombreuses. La recherche s'est systématiquement orientée vers l'élargissement ou le maintien du vivier des diplômés sans s'intéresser également aux itinéraires des diplômés et aux raisons pour lesquelles ils choisissent d'exercer un autre métier.

Les itinéraires complexes que nous avons observés en interrogeant des étudiants suggèrent qu'il s'agit pourtant d'un phénomène massif qui conduit à repenser complètement la notion d'attractivité, soit comme l'attractivité globale d'une formation et de carrières qui prend en compte le contexte de la profession et les raisons qui conduisent à étudier l'ingénierie, soit comme un processus complexe au cours duquel chaque individu construit son propre parcours et croise des formations et des expériences professionnelles dans plus d'un seul domaine. Les parcours de moins en moins linéaires que nous avons observés chez les étudiants donnent de bonnes raisons de penser que le second modèle, celui d'un parcours complexe et individualisé, et par construction, plus interdisciplinaire, est plus proche des parcours effectifs des étudiants d'aujourd'hui.

Repenser l'éducation des ingénieurs en relation avec les formations et leur architecture en terme de disciplines enseignées, d'approches pédagogiques, de cohérence entre les différents enseignements, d'articulation au monde professionnel et social, ce que proposait le colloque de Créteil, conduit à s'interroger sur l'identité actuelle des ingénieurs, ou plutôt sur leurs identités et la manière dont ces identités changent au cours du temps.

En effet, les cultures de l'ingénierie varient d'un pays à l'autre et parfois à l'intérieur d'un même pays comme l'ont montré les auteurs qui ont travaillé sur l'histoire et les identités des ingénieurs (Grelon, Gouzevitch, \& Karvar, 2004) (Giré, Béraud, \& Dechamps, 2000). Les identités changent également d'un domaine et d'une spécialité à l'autre. Certaines spécialités comme l'architecture, l'agronomie, le commerce, « l'ingénierie généraliste ${ }^{6}$ sont considérées comme relevant de l'ingénierie dans certains pays, pas dans d'autres.

Les résultats d'HELENA montrent également que si les hiérarchies et le prestige sont parfois un élément identitaire important, leur importance est plus ou moins marquée. Dans certains pays comme la France, l'ingénierie est plutôt un choix d'excellence qui conduit à des positions managériales, dans d'autres pays, les ingénieurs sont nettement plus focalisés sur des problèmes scientifiques et techniques.

\footnotetext{
${ }^{6}$ Nous faisons ici référence à une caractéristique souvent revendiquée dans les formations d'ingénieurs française, celle de ne pas former à une spécialité ou à un domaine particulier, mais de former à l'ingénierie en général, de donner une formation « polytechnique ». A cette idée est liée la figure de «l'ingénieur généraliste » qui est plus proche d'un manager que d'un expert sur des questions techniques spécifiques. Voir (Dechamps, Giré, \& Béraud, 2000).
} 
Dans un même contexte national, les différences de niveau de diplôme, master et/ou licence, de prestige entre des formations d'excellence et les seconds choix, de hiérarchie entre les écoles, entrainent en réalité une très grande variété des identités derrière la même appellation d' «ingénieur ». Ces variations étaient sensibles dans les études de cas françaises à travers les entretiens menés avec les étudiants des différentes écoles de l'échantillon.

\subsection{Interdisciplinarité et diversité des parcours de formation}

Cette variété des identités et des traditions explique combien le curriculum et les stratégies pédagogiques peuvent être très différents selon les cas. Les motivations et les profils des étudiants sont si différents qu'il est difficile de les comparer et de faire des recommandations générales pour toutes les écoles. Pour aborder de manière exhaustive la question de l'interdisciplinarité, il conviendrait de préciser dans quelle mesure l'interdisciplinarité affecte ces différents contextes et de la décliner selon différents profils. ${ }^{7}$

Plus généralement, à partir de cette variété des identités, des stratégies et des parcours, les résultats d'HELENA appellent à repenser les itinéraires pour devenir ingénieur. Les étudiants ont des parcours de moins en moins linéaires, ce dont nos méthodologies rendent mal compte. Les entretiens ont font apparaître une forte demande de flexibilité et de mobilité qui laisse penser, qu'au-delà d'enseignements de sciences humaines et sociales et d'une interdisciplinarité assumée et réfléchie dans l'offre de formation, la diversité des parcours possibles et les options sont un élément d'attractivité très important pour répondre à cette demande des étudiants.

Pour comprendre à la fois la complexité de l'architecture des formations et la complexité des parcours étudiants, nous devons donc modifier profondément notre approche méthodologique qui reste centrée sur des parcours linéaires, beaucoup plus simples à étudier.

Les entretiens menés au cours du projet HELENA montrent en effet des stratégies étudiantes diverses. D'une part des stratégies conscientes pour tirer le meilleur parti de l'offre académique, mais aussi des parcours très erratiques dans certains cas.

Les représentations jouent un rôle très important dans ce processus. Pour de nombreux étudiants, ajouter une formation au management (donc des sciences humaines et sociales) à leur formation d'ingénieur est supposé leur éviter le risque de rester englués dans des emplois techniques et leur offrir de meilleures opportunités d'emploi. Cette idée est largement partagée par les étudiants des écoles polytechniques de notre échantillon ${ }^{8}$, elle préoccupe moins les étudiants de l'INSA Lyon au prestige et au caractère généraliste bien mieux établis dans les imaginaires et les hiérarchies réelles ou imaginaires.

Des recherches menées dans des cadres indépendants d'HELENA confirment aussi cette tendance, y compris dans les formations les plus élitistes. Dans une communication au colloque annuel du Centre d'Alembert à Orsay ${ }^{9}$, Jean-Claude Millier (Millier, 2011) mentionne par exemple le cas de l'École Nationale du Génie Rural et des Eaux et Forêts (ENGREF). Dans cette école, la moitié des étudiants en thèse, après un diplôme scientifique à l'Ecole Polytechnique ou à l'École Normale Supérieure, que l'on peut qualifier d'institutions parmi les plus élitistes de l'enseignement supérieur français, choisissent de

\footnotetext{
${ }^{7}$ Les articles réunis dans la partie 2 de ce volume, « Influence du contexte », apportent de nombreux éclairages sur cette question.

8 Il s'agit ici de trois écoles polytechniques (ou plus simplement « polytech ») intégrées à des universités, elles ne recrutent pas systématiquement sur concours après les classes préparatoires et offrent des spécialité dans différents domaines spécifiques. Elles ne forment théoriquement pas d'ingénieurs généralistes et ne jouissent ni du prestige ni de l'ancienneté de l'École Polytechnique, école homonyme mais qui recrute et forme les étudiants de manière complètement différente et nettement plus élitiste.

${ }^{9}$ Communication de Claude Millier, ancien Directeur scientifique de l'ENGREF de 1991 à 2006 : «L'évolution de l'ingénierie publique et ses conséquences pour les programmes de recherches qui y sont liés : le cas des sciences pour l'environnement ». Colloque annuel du Centre d'Alembert les 11 et 12 mai 2011 «La paroles aux sciences humaines et sociales : que nous disent-elles sur les sciences et les technologies aujourd'hui ?». http://www.centre-dalembert.u-psud.fr/archivescolloques/la-paroles-aux-sciences-umaines-et-sociales-que-nous-disent-elles-sur-les-sciences-etles-technologies-daujourdhui/ (consulté le 28 octobre 2014).
} 
faire des doctorats en sciences humaines et sociales ou en économie en pensant que ce choix leur ouvrira les portes des cabinets des ministères et plus tard les postes de direction des plus grandes entreprises.

Si nous rapprochons cette communication de l'échantillon d'HELENA, on constate que la même motivation se traduit sur un mode à peine différent à Polytech Orléans, une formation d'ingénieurs récente à l'Université d'Orléans, il s'agit dans les deux cas d'améliorer ses perspectives de carrière (à des niveaux différents) en articulant sa formation scientifique et technique à une formation complémentaire en sciences humaines et sociales dans une perspective interdisciplinaire. De nombreux étudiants de Polytech Orléans craignent de se trouver cantonnés dans des emplois de techniciens et se tournent vers les sciences humaines et sociales ou le management pour échapper aux postes exclusivement « techniques ». Ils attribuent d'ailleurs les mêmes vertus au fait de passer par des périodes d'études à l'étranger. Il s'agit pour eux d'améliorer leur curriculum vitae en lui ajoutant des expériences dans des domaines qui ne sont pas scientifiques et techniques. La formation pluridisciplinaire qui combine le management à l'ingénierie les a attirés pour cette raison, même si de nombreux étudiants ont reconnu être arrivés à Polytech Orléans après un parcours assez erratique dans lequel le hasard ou les résultats des concours ont joué un rôle important. L'interdisciplinarité dans ce cas les a aidés à donner une cohérence à leur parcours. Le rôle important des représentations du management, de la technologie, etc. mériterait ici d'être étudié plus en détail.

\section{Conclusion et perspectives heuristiques}

A partir du projet HELENA, étudier dans une perspective heuristique l'interdisciplinarité dans les formations d'ingénieurs nous a permis de mettre en évidence certaines pistes réflexions, notamment de questionner leur attractivité et leur identité au regard de la construction des parcours de formation et du degré d'interdisciplinarité de ces formations.

Ces réflexions mettent en relief une situation paradoxale. Alors que l'on observe une tendance à gommer le caractère « aride », « difficile », « peu attractif» des sciences et techniques au profit des sciences humaines et sociales dans les études d'ingénieur et à réduire leur importance dans ces formations ${ }^{10}$ (Béraud et al., 2012), dans le même moment le monde politique et économique souligne la nécessité de former des spécialistes en sciences et technique pour soutenir le développement (Mariano Gago, 2004).

Mais cette étude fournit également des pistes pour sortir de ce paradoxe et invite, dans le cadre d'un colloque sur les sciences humaines dans les formations scientifiques professionnalisantes, à repenser les formations et donc l'identité des ingénieurs à la lumière de cette problématique, à ne plus penser les ingénieurs comme des spécialistes de sciences et techniques mais comme des personnes capables de résoudre des problèmes techniques insérés dans leur contexte économique et social, indissociables de ce contexte ${ }^{11}$. Nous pourrions parler d'ingénieurs du « mode $2 »$, en référence aux travaux de Nowotny et Gibbons (Nowotny et al., 2001) (Gibbons et al., 1994), ou encore de versions contemporaines de l'« honnête homme » (ou femme) de la Renaissance.

La recherche sur le rôle joué par l'interdisciplinarité dans les formations peut donc non seulement aider à construire un nouveau curriculum et une nouvelle identité des ingénieurs, mais aussi proposer des perspectives heuristiques. Elle suppose une réflexion approfondie sur les disciplines, la construction des formations, avec leur cohérence et leurs choix pédagogiques propres, et sur le sens des comparaisons internationales. De nouvelles méthodologies qui prennent appui sur les approches développées dans

\footnotetext{
${ }^{10}$ Ces constatations sont tirées à la fois des résultats quantitatifs et des entretiens du projet HELENA. Voir le volume cité en référence pour l'explicitation détaillée de ces résultats.

11 Cette idée d'une combinaison originale de sciences humaines et sociales et de sciences de l'ingénieur trouve des échos dans d'autres contributions ce recueil, par exemple celles de Charles Lenay et alii autour de la « constitutivité mutuelle de l'Homme et de la technique » ou de Guillaume Carnino sur «l'épistémotechnique ».
} 
les ouvrages sur l'interdisciplinarité restent à développer pour mieux comprendre les dynamiques des formations interdisciplinaires.

Au delà de ses résultats spécifiques en réponse à l'appel d'offre européen qui en était à l'origine, le projet HELENA a servi de point de départ afin de mettre en évidence des directions de recherche à venir.

\section{Références}

Abbott, A. (2001). Chaos of Disciplines (New edition.). University of Chicago Press.

Béraud, A. (2003). A European research on women and Engineering Education (2001-2002). European Journal of Engineering Education, 28(4), 435-451.

Béraud, A., Godfroy, A.-S., \& Michel, J. (Eds.). (2012). Gender and Interdisciplinary Education for Engineers: Formation Interdisciplinaire des Ingénieurs et Problème du Genre. Rotterdam, Boston: Sense Publishers.

Collectif. (1988). Pour une histoire des disciplines scolaires (Vol. 38). Service d'Histoire de l'Education/I.N.R.P.

Creating cultures of success for women engineers - Womeng. (2006). Luxembourg: Office for Official Publications of the European Communities.

Dechamps, P., Giré, A., \& Béraud, A. (2000). Les ingénieurs. Identités en questions. Paris : L'Harmattan.

European Commission. (2010). Europe 2020 A strategy for smart, sustainable and inclusive growth (No. $\operatorname{COM}(2010)$ 2020) (p. 37). Bruxelles. http://ec.europa.eu/eu2020/pdf/COMPLET\% 20EN\%20BARROSO\%20\%20\%20007\%20-\%20Europe\%202020\%20-\%20EN\%20version.pdf (lien consulté le 29/11/14).

Gibbons, M., Limoges, C., Nowotny, H., Schwartzman, S., Scott, P., \& Trow, M. (1994). The New Production of Knowledge: The Dynamics of Science and Research in Contemporary Societies. London?; Thousand Oaks, Calif: SAGE Publications Ltd.

Giré, A., Béraud, A., \& Dechamps, P. (2000). Les ingénieurs. Identités en questions. L’Harmattan. Godfroy, A.-S. (2012). Thinking Interdisciplinarity in Engineering Education: Challenges for Future Research. In Gender and Interdisciplinary Education for Engineers: Formation Interdisciplinaire des Ingénieurs et Problème du Genre (pp. 193-202). Rotterdam, Boston: Sense Publishers.

Grelon, A., Gouzevitch, I., \& Karvar, A. (2004). La formation des ingénieurs en perspective? : Modèles de référence et réseaux de médiation XVIIIe - XXe siècle. PU Rennes.

Heintz, C., Origgi, G., \& Sperber, D. (Eds.). (2004). Rethinking Interdisciplinarity. Retrieved from http://www.interdisciplines.org/medias/confs/archives/archive_3.pdf

INDECS Project Final Report: Potentials of interdisciplinary degree courses in engineering, information technology, natural and socio-economics sciences in a changing society (2002).

Kaptan, K., \& Timurlenk, O. (2012). Challenges for Science Education. Procedia - Social and Behavioral Sciences, 51, 763-771.

Kourilsky, F., \& Tellez, J. (2003). Ingénierie de l'interdisciplinarité. Un nouvel esprit scientifique. Editions L'Harmattan.

Maingain, A., \& Dufour, B. (2002). Approches didactiques de l'interdisciplinarité. De Boeck Université.

Mariano Gago, J. (2004). Europe needs more scientists. Increasing Human Resources for Science and Technology in Europe, Report of the High Level Group on Human Resources for Science and Technology in Europe chaired by Prof. José Mariano Gago (No. ISBN 92-894-8458-6) (p. 215). Luxembourg: European Commission. http://ec .europa.eu/research/conferences/ 2004/sciprof/pdf/final_en.pdf (lien consulté le 8 juillet 2014).

Millier, C. (2011, et 12 mai). L'évolution de l'ingénierie publique et ses conséquences pour les programmes de recherches qui y sont liés? : le cas des sciences pour l'environnement. Presented at the Colloque annuel 2011 du Centre d'Alembert « La paroles aux sciences humaines et sociales ? : que 


\section{SHS Web of Conferences}

nous disent-elles sur les sciences et les technologies aujourd'hui ?, » Orsay, France. http://www . centre-dalembert.u-psud.fr/archives-colloques/la-paroles-aux-sciences-umaineset-sociales-que-nous-disent-elles-sur-les-sciences-et-les-technologiesdaujourdhui/ (lien consulté le 29 octobre 2014).

Nowotny, H., Scott, P., \& Gibbons, M. (2001). Rethinking Science : Knowledge and the Public in an Age of Uncertainty. Polity Press.

Origgi, G., \& Darbellay, F. (2010). Repenser l'interdisciplinarité. Editions Slatkine.

Osborne, J., Simon, S., \& Collins, S. (2003). Attitudes towards science: A review of the literature and its implications. International Journal of Science Education, 25(9), 1049-1079.

Paulsen, F. D. \& T. (2008). Le Défi de l'Inter- et Transdisciplinarité. Concepts, méthodes et pratiques innovantes dans l'enseignement et la recherche. Presses Polytechniques et Universtaires Romandes.

Thaler, A. (2012). InterdIscIplInarItIes - students' perception of interdisciplinary engineering education in Europe. In Gender and Interdisciplinary Education for Engineers: Formation Interdisciplinaire des Ingénieurs et Problème du Genre (pp. 203-215). Rotterdam, Boston: Sense Publishers.

Vinck, D. (2001). Pratiques de l'interdisciplinarité ? : Mutations des sciences, de l'industrie et de l'enseignement. Grenoble: PU Grenoble.

Waechter, C. (2005). A Gendered Look at Interdisciplinarity in Engineering Education. In A. Bammé, G. Getzinger, \& B. Wieser (Eds.), Yearbook 2005 of the Institute for Advanced Studies on Science Technology and Society (pp. 469-493). Munich et Vienne: Bammé, Getzinger, Wieser.

Waechter, C. (2012). Interdisciplinary teaching and learning for diverse and sustainable engineering education. In Gender and Interdisciplinary Education for Engineers: Formation Interdisciplinaire des Ingénieurs et Problème du Genre (pp. 51-67). Rotterdam, Boston: Sense Publishers. 